\title{
The effects of training mental health practitioners in medication management to address nonadherence: a systematic review of clinician-related outcomes
}

\author{
This article was published in the following Dove Press journal: \\ Nursing: Research and Reviews \\ 27 May 2013 \\ Number of times this article has been viewed
}

\author{
Daniel Bressington' \\ Esther Coren' \\ Douglas Maclnnes ${ }^{2}$ \\ 'Department of Health, Well-Being \\ and Family, ${ }^{2}$ Centre for Health and \\ Social Care Research, Canterbury \\ Christ Church University, \\ Canterbury, UK
}

Correspondence: Daniel Bressington Department of Health, Well-Being and Family, Canterbury Christ Church University, North Holmes Road, Canterbury, Kent CTI IQU, UK Tel +44 I 227782 6II

Email daniel.bressington@canterbury. ac.uk
Background: Nonadherence with medicine prescribed for mental health is a common problem that results in poor clinical outcomes for service users. Studies that provide medication management-related training for the mental health workforce have demonstrated that improvements in the knowledge, attitudes, and skills of staff can help to address nonadherence. This systematic review aims to establish the effectiveness of these training interventions in terms of clinician-related outcomes.

Methods: Five electronic databases were systematically searched: PubMed, CINAHL, Medline, PsycInfo, and Google Scholar. Studies were included if they were qualitative or quantitative in nature and were primarily designed to provide mental health clinicians with knowledge and interventions in order to improve service users' experiences of taking psychotropic medications, and therefore potentially address nonadherence issues.

Results: A total of five quantitative studies were included in the review. All studies reported improvements in clinicians' knowledge, attitudes, and skills immediately following training. The largest effect sizes related to improvements in clinicians' knowledge and attitudes towards nonadherence. Training interventions of longer duration resulted in the greatest knowledge- and skills-related effect sizes.

Conclusion: The findings of this review indicate that training interventions are likely to improve clinician-related outcomes; however, due to the methodological limitations of the current evidence base, future research in this area should aim to conduct robust randomized controlled trials with follow-up and consider collecting qualitative data to explore clinicians' experiences of using the approaches in clinical practice.

Keywords: staff training, treatment adherence, medication management

\section{Background}

Nonadherence with medications prescribed for mental health is a common problem that results in poor outcomes for service users. Complete or partial nonadherence with treatment is associated with increases in relapse rates, more hospital admissions, and higher rates of suicide. ${ }^{1-3}$ In response to this clinical need, numerous research projects have evaluated the efficacy of clinical psychosocial interventions designed to improve adherence with and response to psychotropic medication. The results of these studies have been mixed, but findings have demonstrated the potential of such approaches to improve service users' adherence with treatment, resulting in meaningful clinical gains, such as improved levels of symptoms and 
reduced hospitalization rates. ${ }^{4-6}$ The most promising of these efficacy studies was a randomized controlled trial of "compliance therapy" versus "nonspecific counseling" conducted by Kemp et al. ${ }^{4}$ The study involved 74 patients with a psychotic illness; the results showed that in comparison with the control group, the patients who received four to six sessions of compliance therapy had improved insight into their illness, better attitudes towards treatment, reduced rates of readmission to hospital, and increased levels of treatment adherence, both in the short term and at 18-month follow-up.

One major criticism of the intervention studies designed to enhance adherence with treatment is that the interventions are often delivered by expert practitioners, and that the possible benefits may not be generalized to mental health workers who help to manage medication as part of routine practice. ${ }^{7}$ As a result, a number of studies have aimed to evaluate the outcomes of training the mental health workforce to incorporate such interventions into everyday clinical situations. These studies have reported a number of consistently positive outcomes for service users, particularly in relation to reductions in symptoms, improvements in attitudes towards treatment, enhanced quality of therapeutic alliance, and improved levels of adherence with medication. ${ }^{8,9}$ Previous research has also demonstrated that there is a relationship between clinician knowledge and attitudes relating to medication management and ability to address nonadherence issues. ${ }^{10}$

Due to this body of evidence, research programs have been conducted that report the results of medicationmanagement training interventions in terms of clinicianrelated outcomes. These programs report variations in terms of the duration and content of teaching, which have financial costs and educational implications. To date there are no published systematic reviews that compare the results of these programs. A review would therefore establish the efficacy of such programs for mental health practitioners in order to inform the development and refinement of future medication management educational packages.

\section{Methods}

\section{Review questions}

Which medication management related staff training programs have investigated the impact of training on clinician-related outcomes? What are the effects of medication-management training programs on mental health clinicians' knowledge, attitudes, skills, and clinical practice?

\section{Study inclusion criteria}

Studies were included if they met the following criteria:

- Participants in the studies were mental health clinicians (from any professional background) who work with service users in managing medication for mental health problems.

- The training interventions were primarily designed to provide mental health clinicians with knowledge and interventions in order to improve service-users' experiences of taking psychotropic medications, and therefore potentially address nonadherence issues.

- Outcomes reported in the studies related to clinicians' knowledge, attitudes, skills, or confidence in managing medications for mental health.

- The studies were either qualitative studies or quantitative primary research, quasiexperimental, or experimental studies that reported clinician-related outcome measures.

- The studies were written in English and published between January 2000 and June 2012 (these were included in order to capture the contemporary issues relating to the publication of results for therapeutic approaches to address nonadherence and for ease of contrasting and comparing results).

\section{Search strategy}

Search terms were entered into five databases (PubMed, CINAHL, Medline, PsycINFO, and Google Scholar). Reference lists from published work were also hand-searched to identify potential relevant studies, and recent key researchers were contacted to enquire about potential gray literature. The search strategy is detailed in Table 1.

\section{Study-quality assessment}

The studies included in this review were assessed for their quality using the Critical Appraisal Skills Programme (CASP) tool. ${ }^{11}$ As none of the studies identified were randomized, controlled trials, selected elements of the most closely related CASP cohort study-appraisal tool were used to determine study quality (please see Table 2 for the criteria used). Two of the researchers independently scored the studies before meeting to discuss and agree on a consensus score. Each study was awarded a potential score from a maximum of 14; the minimum cutoff score for inclusion in this review was 8 . Studies scoring $8-11$ were defined as medium quality, and studies scoring 12 or over were classified as being of good quality. ${ }^{12}$ The exclusion of studies with a score of $<8$ 
Table I Search strategy

\begin{tabular}{ll}
\hline Search line number & Search terms \\
\hline 1 & $\begin{array}{l}\text { Training/in-service training } \\
\text { Education, professional/education/nursing } \\
\text { education research/education, nursing } \\
\text { Compliance/de (drug effects) }\end{array}$ \\
3 & Medication adherence/adherence \\
4 & Professional-patient relations/alliance \\
5 & Satisfaction \\
6 & Drug therapy/medication management \\
7 & Psychopharmacology \\
8 & Health knowledge, attitudes, practice/staff \\
9 & knowledge \\
10 & Clinical competence/staff skills \\
11 & I or 2 \\
12 & 3 or 4 or 5 or 6 \\
13 & 7 or 8 or 9 or I0 \\
14 & Mental health/psychiatry \\
15 & II and I2 and I3 and I4 \\
16 & Remove duplicates from I5 \\
\hline
\end{tabular}

was intended to reduce the amount of bias associated with methodological issues and improve comparability.

\section{Data synthesis}

In order that the outcomes of the various studies could be compared and contrasted, the standardized mean differences (SMDs) and the 95\% confidence intervals (CIs) for each area of study outcome were calculated using RevMan 5.1 (Nordic Cochrane Centre, Copenhagen, Denmark) software. If the 95\% CI of the SMD crossed zero, this indicated a nonstatistically significant effect. We used Cohen's widely adopted criteria to determine the relevance of effect sizes $(0.2=$ small, $0.5=$ moderate, $0.8=$ large $).{ }^{13}$ Due to the apparent high

Table 2 CASP study quality criteria checklist items

\begin{tabular}{ll}
\hline Question & Scoring \\
\hline Was the cohort recruited in an & Yes (2), can't tell (I), \\
acceptable way? & no $(0)$ \\
Was the exposure accurately measured to & Yes (2), can't tell (I), \\
minimize bias? & no (0) \\
Was the outcome accurately measured to & Yes (2), can't tell (I), \\
minimize bias? & no $(0)$ \\
Have the authors identified all important & Yes (2), can't tell (I), \\
confounding factors? & no (0) \\
Have they taken account of the confounding & Yes (2), can't tell (I), \\
factors in the design and/or analysis? & no (0) \\
Was the follow-up of subjects complete & Yes (2), can't tell (I), \\
enough? & no (0) \\
Was the follow-up of subjects long enough? & Yes (2), can't tell (I), \\
& no (0) \\
\hline
\end{tabular}

Adapted from Critical Appraisal Skills Programme (CASP). Making sense of evidence about clinical effectiveness. Available from: http://www.casp-uk.net/wp-content/ uploads/201 I/I I/CASP Cohort Appraisal Checklist 14oct 10.pdf." degree of heterogeneity in terms of study designs, outcome measures, and length/content of the training interventions, it was not appropriate to conduct meta-analysis. ${ }^{14}$

\section{Results}

Figure 1 shows the results of the literature search within a PRISMA (preferred reporting items for systematic reviews and meta-analyses) flow diagram. ${ }^{15}$ Initially, 91 potential studies were identified; screening the abstracts and titles narrowed down the number of studies to a potential 20. Of these, 15 were excluded. Reasons for excluding studies included not being primary research, educational intervention not mental health-related, and the main focus of training not being on managing treatment with medication. No relevant qualitative studies were identified, and therefore this review includes only studies reporting quantitative data. Table 3 details the study characteristics, CASP quality scores and results of the studies included in the review.

\section{Participants}

The five studies included in this review were carried out in a variety of international settings. Two studies (Byrne et $\mathrm{al}^{16}$ and Byrne and Deane ${ }^{9}$ ) were conducted in Australia, two were carried out in the UK (Gray et $\mathrm{al}^{17}$ and Surguladze et $\mathrm{al}^{18}$ ), and one was conducted in the US (Byrne et $\mathrm{al}^{19}$ ).

In three studies, ${ }^{9,16,17}$ the clinicians involved worked in community mental health settings, one study investigated inpatient mental health clinicians, ${ }^{19}$ while the Surguladze et $\mathrm{al}^{18}$ study reports that around $60 \%$ of participants worked in adult psychiatry inpatient environments (and the remainder within community teams).

The professional background of clinicians also varied across the studies; all participants in the Gray et a ${ }^{17}$ study were registered mental health nurses, and all the clinicians trained by Surguladze et a $1^{18}$ were psychiatry trainees. The majority of clinicians in the three studies by Byrne were nurses; however, a range of other professional groups were also involved, including social workers, technicians, psychologists and allied health professions.

\section{Study designs}

All the studies included in this review were uncontrolled studies that utilized a pre- and postintervention design to explore the impact of training. Surguladze et $\mathrm{al}^{18}$ also gathered data from 54 psychiatrists that did not attend the program, as a comparison group. Four of the studies included in this review report primarily on clinician-related outcomes, while Byrne and Deane ${ }^{9}$ report both clinician- and service user-related outcomes. 


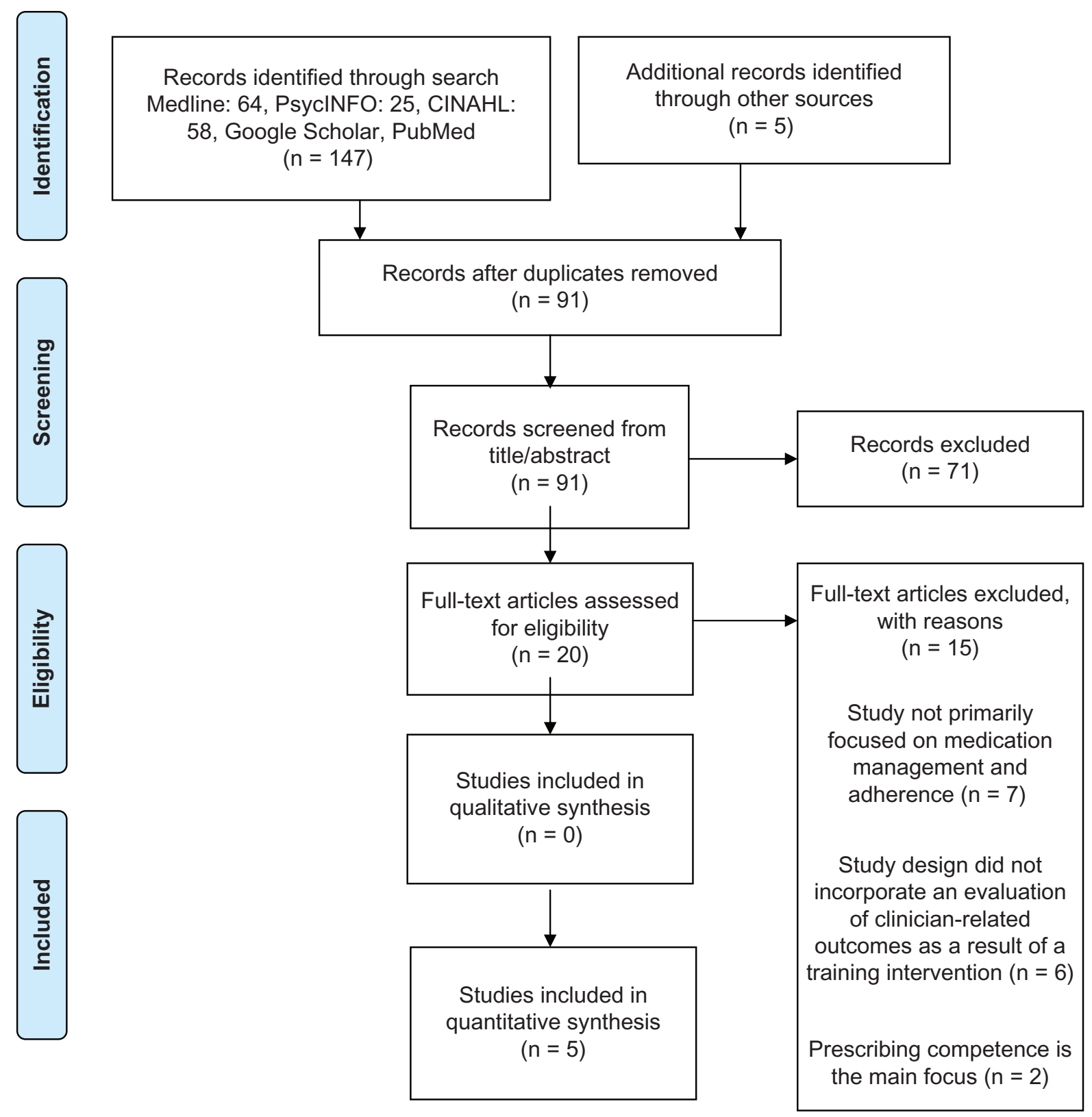

Figure I PRISMA (preferred reporting items for systematic reviews and meta-analyses) flow diagram.

Only one study (Byrne and Deane) ${ }^{9}$ used a design that measured the durability of any changes in knowledge, skills, or attitudes. They reported outcomes immediately posttraining, and at both 6 and 12 months. All the other studies measured post-training outcomes immediately after the teaching was delivered. The follow-up period for Gray et al ${ }^{17}$ was effectively 10 weeks (immediately after the program finished).

\section{Outcome measures}

The outcome measures used in the studies included in this review also varied considerably. They were designed to measure one or a combination of skills, attitudes, and knowledge related to working with service users who have difficulty in adhering to medication regimens.

Surguladze et a ${ }^{18}$ developed a clinician-rated questionnaire with 35 statements grouped into six subscales: causes of noncompliance, costs of noncompliance, rating of important skills, confidence in using skills, beliefs about managing mental illness, and attitudes towards patient compliance. The researchers do not mention how this questionnaire was devised or whether any psychometric properties had been established. Overall pre- and post-training means and standard deviations were not reported in the study. 


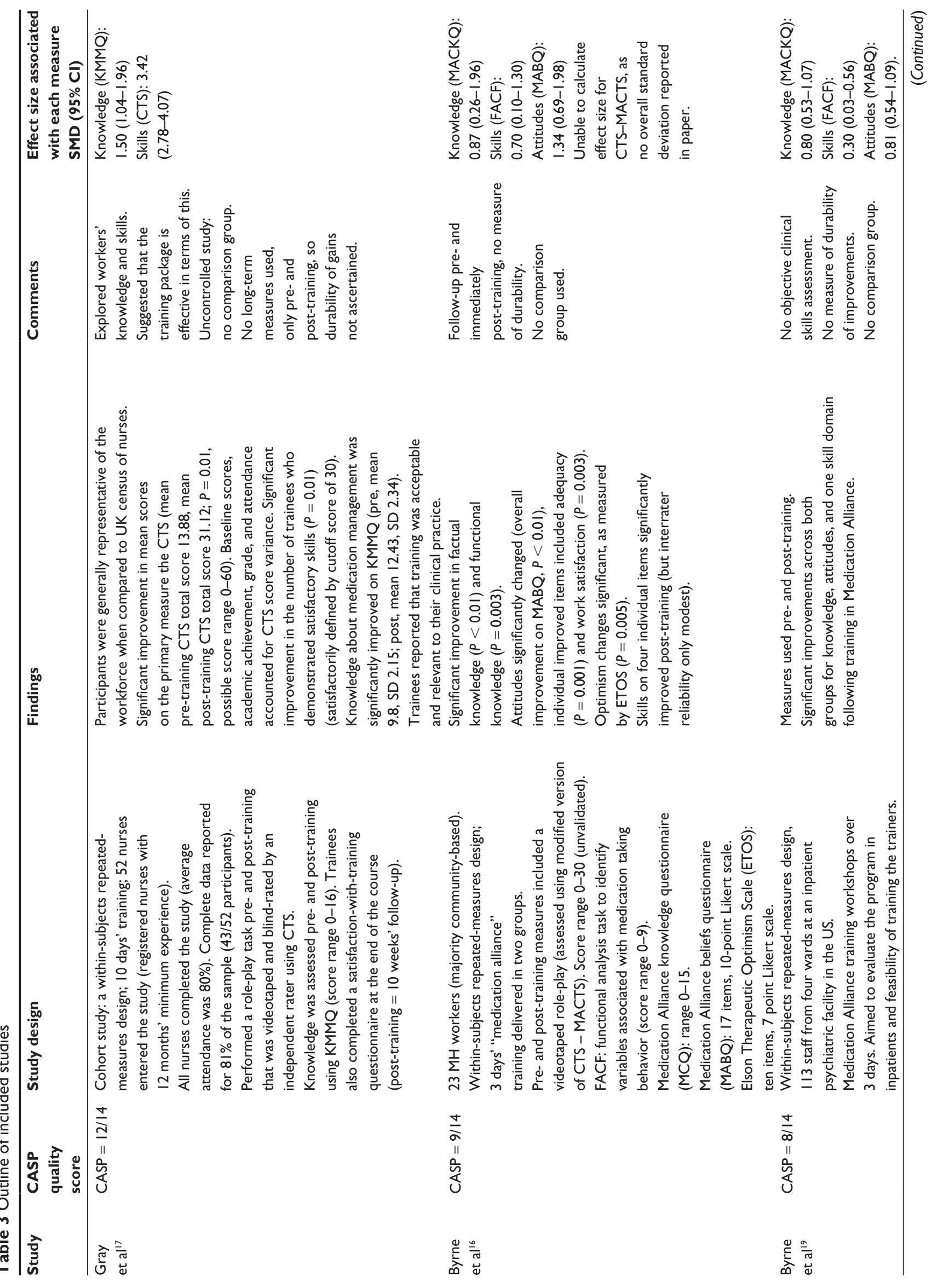




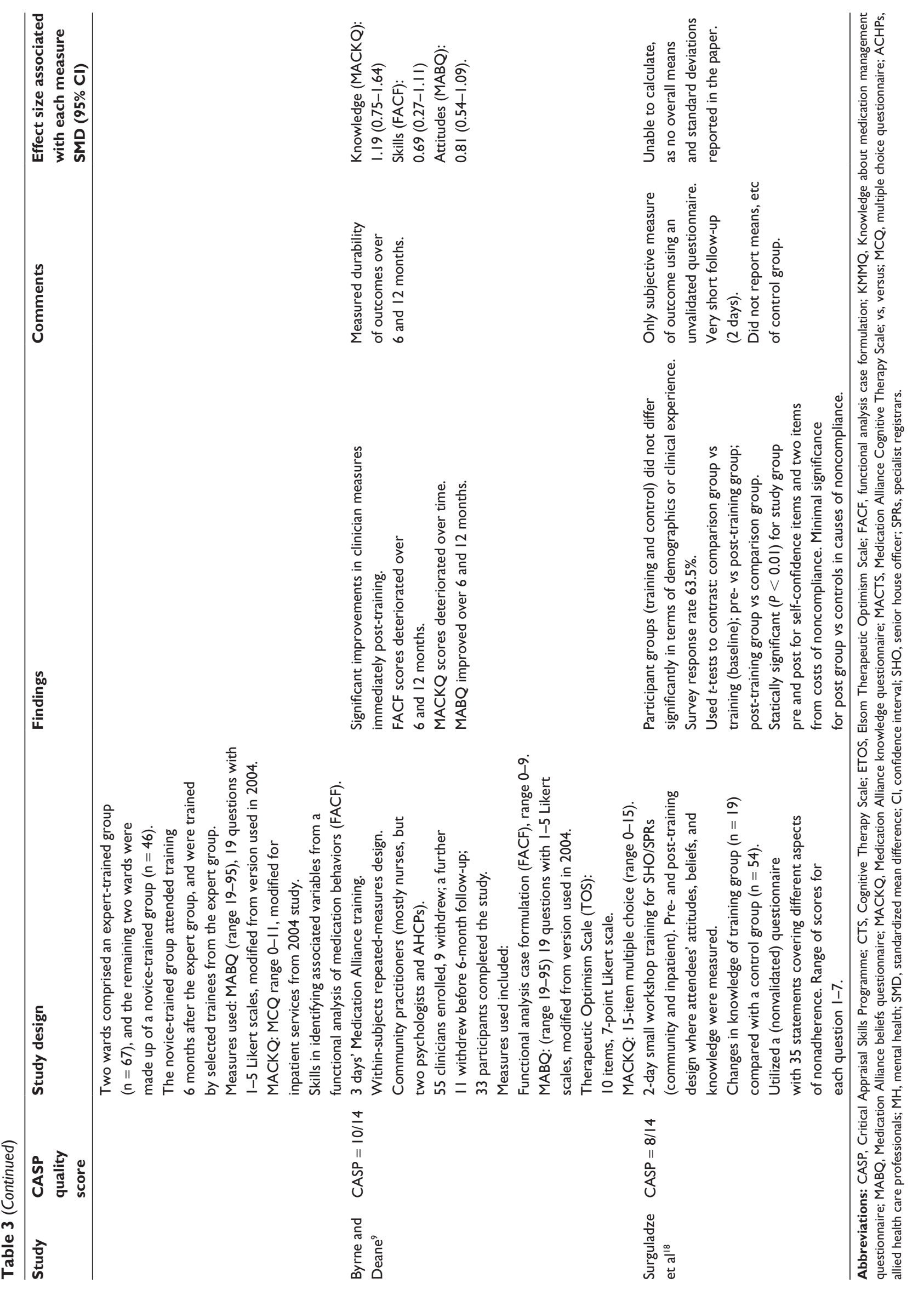


The Gray et al ${ }^{17}$ study utilized a modified version of the Cognitive Therapy Scale (CTS), ${ }^{20,21}$ which was used to blindrate a role-play pre- and post-training. The modified CTS is a valid and reliable measure of clinicians' skills and consists of ten items rated on a 7-point scale. Knowledge was assessed at the same time points using a 16-item multiple-choice knowledge about medication management questionnaire, ${ }^{17}$ which was designed by the researchers. This questionnaire was designed to have content validity by basing the questions on the course content, and the test-retest reliability was established in a group of nurses not involved in the project $(P<0.05)$. Participants also completed a satisfaction-withtraining questionnaire.

The first study by Byrne et al, ${ }^{16}$ published in 2004, utilized a range of measures designed to assess the skills, knowledge, and attitudes of staff towards working with nonadherent service users. The outcome measures used in the study were all developed by the researchers. Similarly to the study by Gray et al, ${ }^{17}$ clinician skills in a 15 -minute videoed role-play were blind-rated using a modified version of the CTS; $;^{20,21}$ the researchers called this measure the Medication Alliance and Cognitive Scale for Psychosis, and total scores ranged from 0 to 30 . Byrne et al ${ }^{16}$ also measured clinicians' ability to identify variables associated with medication-taking behavior from a case vignette using a functional analytic case formulation (FACF) assessment, which had a range of scores from 0 to 9 . Clinicians' knowledge was assessed using the Medication Alliance clinician knowledge questionnaire (MACKQ), which is a 15 -item multiple-choice questionnaire. The beliefs of clinicians were measured using the Medication Alliance beliefs questionnaire (MABQ) which has 17 items rated on a scale from 1 (extremely difficult) to 10 (very easy), and was also developed by the researchers and loosely based on the alcohol and alcohol problems perception questionnaire. ${ }^{22,23}$ The attitudes of staff in terms of their optimism in working with service users were explored using the Elsom Therapeutic Optimism Scale (ETOS), ${ }^{24}$ which is a 10 -item measure with each item rated on a 7-point Likert scale. The researchers report that the ETOS has acceptable internal reliability and test-retest reliability.

The Byrne et al study published in $2010^{19}$ also utilized the FACF and MACKQ. The MACKQ used in this study was reduced to 11 items, as 4 items in the original questionnaire did not relate to inpatient settings. The researchers further adapted the MABQ to include a total of 19 items on a scale of 1 (strongly disagree) to 5 (strongly agree), and reported that the internal reliability of the measure was good.
Byrne and Deane ${ }^{9}$ used the FACF, MABQ, ETOS, and MACKQ, which had been used in earlier studies. For the purposes of this review, we excluded the results from the ETOS from the analysis, as the study did not report pre- and post-training scores or standard deviations. The pretraining scores and standard deviations for the FACF, MABQ, and MACKQ were obtained from the first author by means of personal communication.

Despite most studies collecting demographic details of clinicians, only one study (Gray et al) ${ }^{17}$ explored potential predicators of variations in clinician outcomes; they performed an exploratory linear regression analysis to determine that level of previous academic achievement, clinical grade, baseline scores, and attendance at training predicted improved clinical skills.

\section{Training interventions}

The duration of the training interventions varied considerably across the studies: the three studies with Byrne as lead author used a 3-day Medication Alliance training program that was delivered in one block, Gray et al ${ }^{17}$ provided 10 days' "medication management" training, while Surguladze et al's "compliance therapy" lasted only 2 days. ${ }^{18}$

The content of the teaching programs also differed significantly; despite all the studies using elements of "compliance therapy," which was pioneered by Kemp et al, ${ }^{4}$ each project placed particular emphasis on different issues. Surguladze et al ${ }^{18}$ taught psychiatric trainees a combination of cognitive therapy techniques, motivational interviewing, and psychoeducation that comprised "compliance therapy."

Compliance therapy comprises a combination of motivational interviewing and cognitive-behavioral interventions. ${ }^{4}$ The therapeutic approach consists of three distinct phases, which are designed to engage patients in collaborative conversations about their treatment while avoiding confrontation. The first phase reviews the patient's illness history so that their stance towards treatment can be elicited. The second stage explores patients' ambivalence about medication, and stage three focuses on maintaining long-term treatment.

Gray et $\mathrm{al}^{17}$ combined compliance therapy skills, assessment skills, psychopharmacology knowledge, and clinical supervision sessions to form a 10-day "medication management" training package that was delivered over a 10 -week period. The psychopharmacology and assessment content comprised almost $50 \%$ of the overall program, and was intended to help improve clinicians' confidence when exchanging information with patients about their treatment. This content covered the mechanisms of action of commonly 
prescribed psychotropic drugs, common side effects, and longer-term safety considerations. Clinicians were also taught how to assess and manage the common side effects of treatment, and use standardized rating scales to assess psychopathology. The emphasis on providing clinical supervision as part of the training package differentiates the content of this teaching program from the other studies; participants were able to discuss their current work with service users who had adherence problems, and this may have had some influence on the learning outcomes. This is also the only study in the review that specifically taught psychopharmacology and trained clinicians to use assessment tools.

Byrne's Medication Alliance 3-day programmes ${ }^{9,16,19}$ were based on some elements of compliance therapy and did not cover any psychopharmacology. The main focus of the program was to improve the therapeutic relationship between service user and clinician so that beliefs and attitudes associated with taking medication were able to be explored and modified. The training focused more heavily on using a cognitive-behavioral case formulation by utilizing a functional analysis approach in order to ensure that interventions were individualized and tailored to address issues raised by service users. The Medication Alliance teaching package also incorporated relapse-prevention approaches and the use of the stress-vulnerability model of psychosis to explain etiology. Clinical supervision (or clinical coaching) sessions were offered on a monthly basis for 6 months following training as part of the most recent Byrne et al ${ }^{19}$ study; however, the participants' attendance at the sessions was variable (Byrne, personal communication, 2012).

\section{Effects of the training interventions}

The forest-plot graphs for each area of outcome are presented in Figures 2-4 and show the effect sizes (SMD) and 95\% CIs for each of the individual studies included in this review. The overall effect size for the Surguladze et al ${ }^{18}$ study could not be calculated, as the pre- and post-training total means and standard deviations were not reported; however, the researchers reported statistically significant improvements post-training on seven of the 28 items of the questionnaire. The areas where most improvements were observed related to the self-confidence of clinicians in dealing with nonadherence issues. The overall effect size for skills as measured by the Medication Alliance and Cognitive Scale for Psychosis in Byrne et $\mathrm{al}^{16}$ likewise could not be calculated, as the study did not report the total mean and standard deviation.

Where individual effect sizes could be calculated, the clinician outcomes can be categorized as relating to knowledge, skills, and attitudes. The predominant areas of clinician knowledge that were measured were predictors of nonadherence, prevalence of nonadherence, and awareness of the causes and consequences of nonadherence. Some examples of the attitudes that were assessed included clinicians' ideas about patients' perceived ability to collaborate in decisions about treatment, the rights of patients to refuse treatment, and the feelings evoked when dealing with clinical nonadherence. The focus of skills assessment was on clinicians' ability to listen empathically, build collaborative therapeutic relationships, and avoid persuasion during conversations about taking medication.

\section{Knowledge}

Figure 2 shows the knowledge-related effect sizes of each study. All the studies resulted in large effect sizes; however, the largest effect size was demonstrated by the Gray et al ${ }^{17}$ study (1.50, 95\% CI 1.04-1.96).

\section{Skills}

Figure 3 details the skills-related effect sizes. Similarly to the knowledge-related outcomes, the largest effect size was associated with the Gray et a ${ }^{17}$ training program $(3.42,95 \%$ CI 2.78-4.07). The other three studies authored by Byrne all showed medium effect sizes in terms of clinicians' skills.

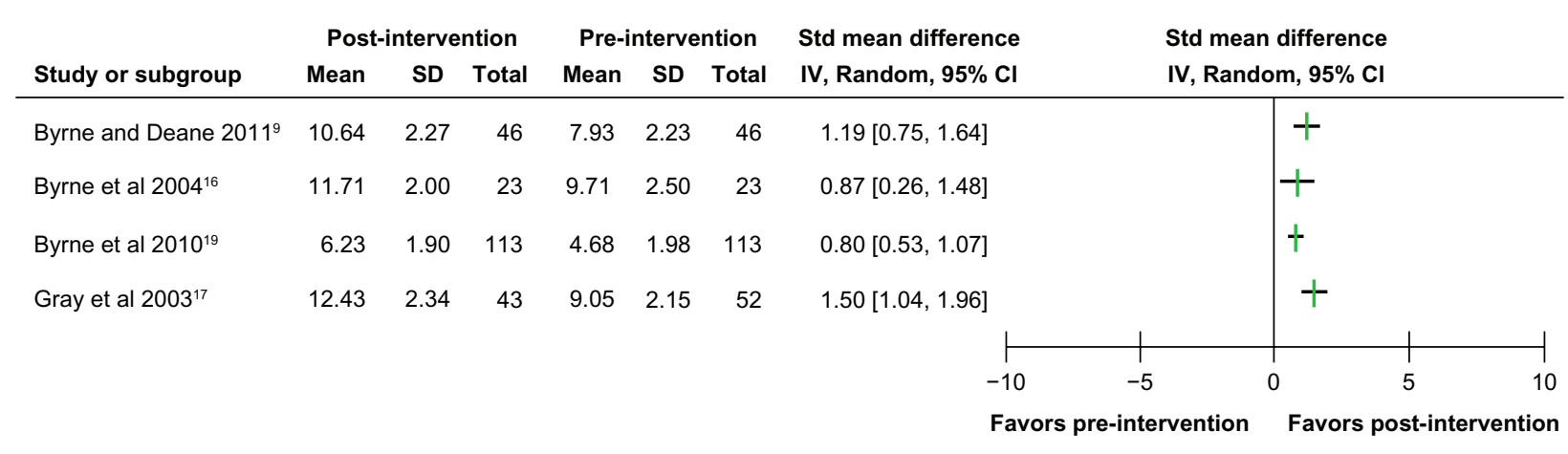

Figure 2 Outcome - clinicians' knowledge.

Abbreviations: SD, standard deviation; IV, inverse variance; $\mathrm{Cl}$, confidence interval; Std, standardized. 


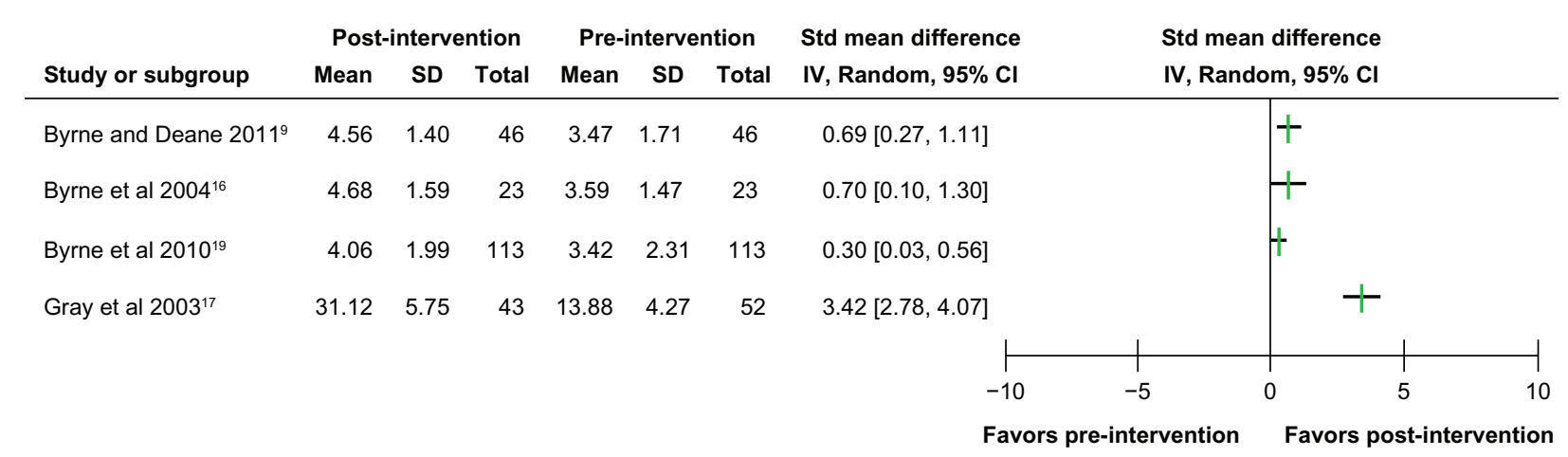

Figure 3 Outcome - clinicians' skills.

Abbreviations: SD, standard deviation; IV, inverse variance; $\mathrm{Cl}$, confidence interval; Std, standardized.

However, direct comparisons of skill-related effect sizes between the Gray et al and Byrne et al studies are not possible, because the Gray et $\mathrm{al}^{17}$ study utilized an observed structured clinical examination approach, while Byrne et al ${ }^{10,19}$ assessed participants' ability to identify causes of nonadherence in a fictitious case study.

\section{Attitudes}

The effect sizes for clinicians' attitudes are presented in Figure 4. All the studies resulted in large effect sizes, with the largest effect size $(1.34,95 \%$ CI $0.69-1.98)$ reported by Byrne et al. ${ }^{16}$

\section{Discussion}

All the studies in this review were uncontrolled studies, and as such we cannot definitively attribute changes in clinician's knowledge, attitudes, and skills to the training programs. The degree of heterogeneity in terms of study populations, length of the training interventions, study-outcome measures, and study designs complicates direct comparisons of results. However, this literature review has gathered the best evidence available in English in order to define the effects of medication-management training on clinician outcomes immediately post-training.
Despite the methodological shortfalls of the included studies, it is possible that there is an association between the observed clinician-related improvements and the educational interventions. The Surguladze et a ${ }^{18}$ study reinforces this conclusion, as it compares outcomes with a cohort of clinicians who did not receive any training, and identifies significant differences between the two groups. All studies reported improvements across the three areas of outcome, and none of the studies reported negative results in any of the clinician-outcome domains.

The majority of outcome measures used in the studies has been constructed for the specific purposes of the research; however, some of the researchers made concerted attempts to establish their psychometric properties. All the studies in this review used multiple-choice questionnaires to assess knowledge acquisition and measure changes in attitudes towards nonadherence. The validity and reliability of this strategy may be doubtful; one of its potential limitations is that it may encourage clinicians' rote recall of facts rather than encouraging engagement in meaningful learning. ${ }^{25} \mathrm{~A}$ strategic approach to learning would be more likely when participants are aware that the same multiple-choice questionnaires will be repeated in order to assess outcomes; participants may remember the questions they were asked before the training

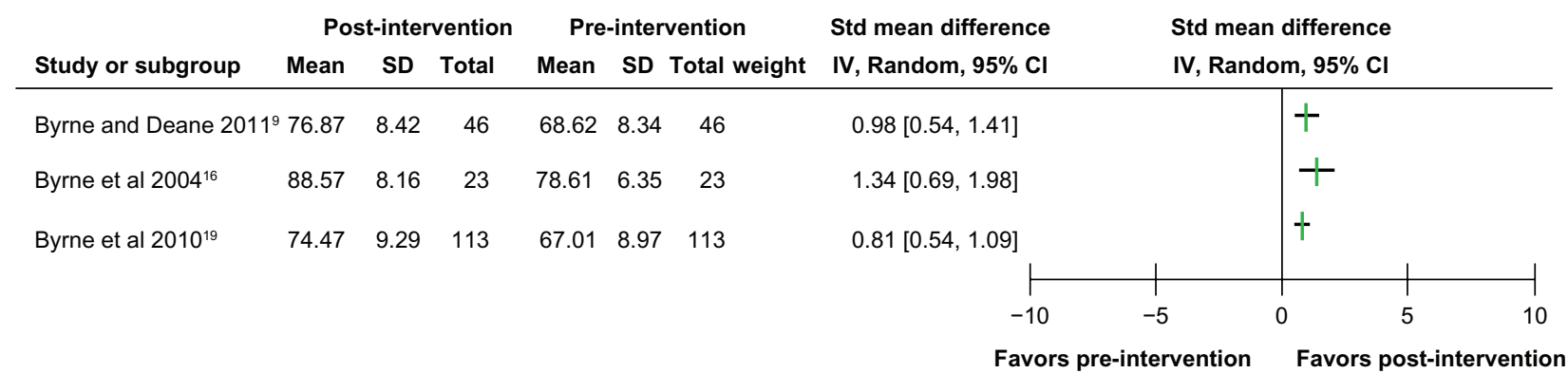

Figure 4 Outcome - clinicians' attitudes.

Abbreviations: SD, standard deviation; IV, inverse variance; $\mathrm{Cl}$, confidence interval; Std, standardized. 
and therefore memorize the answers accordingly. This also provides a potential explanation as to why the greatest effect sizes are observed in relation to knowledge and attitudes.

There appears to be a relationship between the duration of training and effects of training on knowledge and skills; the Gray et $\mathrm{al}^{17} 10$-day training program resulted in the largest effect sizes of all the studies across both these domains. Although the knowledge-effect size of Gray et al's study (1.50, 95\% CI 1.04-1.96) is greater than that of Byrne and Deane $^{9}(1.19,95 \%$ CI $0.75-1.64)$, this only equates to one additional point on a 16-item multiple-choice questionnaire, and therefore the benefit of this additional gain for significantly increased training costs is doubtful.

It is possible that the provision of clinical supervision (or clinical coaching) in the Gray et $\mathrm{al}^{17}$ and Byrne et al ${ }^{19}$ studies could also have an impact on what clinicians remember from taught sessions and how they apply theory to clinical practice. The use of reflection to enhance critical thinking about clinical practice and improve learning has been reported by a number of researchers. ${ }^{26-28}$ However, Byrne (personal communication, 2012) confirmed that there were no statistically significant differences in outcomes for those clinicians involved in the 2010 study ${ }^{19}$ who attended coaching sessions, compared to those who did not.

Only one of the studies (Byrne and Deane) ${ }^{9}$ reported follow-up data to establish whether improvements were maintained over an extended period. The results showed that skills (FACF) and adherence knowledge (MACKQ) decreased over time, while general attitudes (MABQ total) improved at both 6 and 12 months. Therefore, it is possible that the other studies may overestimate the potential effects of training on clinical practice; measuring changes directly after training when the content is fresh in the participants' minds is likely to measure how well the content is recalled, rather than how it is understood and applied to practice.

Clinicians' attitudes appear to be responsive to educational interventions, as they were found to significantly improve immediately post-training, with large effect sizes observed in all three of Byrne's studies. It is also possible that these gains may be maintained over time, because as reported by Byrne and Deane, ${ }^{9}$ attitudes may continue to change in a positive direction following the use of the approaches in clinical practice.

\section{Implications for practice and education}

Knowledge, skills, and attitudes appear to improve significantly as a result of medication management-related training interventions. Training interventions delivered over a longer duration and those with mechanisms built in for clinical supervision seem to result in greater knowledge-related effect sizes than shorter programs. Clearly, delivering a longer training course will have negative cost implications, and the results from this review suggest that the additional educational gains may not justify the extra costs. Future medication-management training programs could consider offering a shorter duration of training that is delivered over a longer time span (ie, 1 day per week for 3 or 4 weeks), as this approach would also provide the opportunity to discuss the clinical application of approaches during clinical supervision provision that is integrated into the teaching program. The deterioration in knowledge and skills over time reported by Byrne and Deane ${ }^{9}$ suggests that ongoing educational top-up sessions may be useful in order to improve the durability of positive outcomes.

\section{Implications for further research}

The most important implication for future research arising from this review is the need for robustly conducted randomized controlled trials with an active control group so that clinicians' improvements can be more certainly attributed to the training intervention. The evidence surrounding the durability of improvements as a result of training is also minimal; therefore, future studies should aim to adopt a longitudinal approach in order to ascertain how well improvements are maintained over time.

None of the studies reviewed explored from a clinician's perspective how the knowledge and skills learned during training had been applied in clinical practice, and therefore it is difficult to ascertain which elements of training clinicians perceive as being the most beneficial and what barriers to implementation they encountered when applying interventions. In general, there is a paucity of published qualitative data, which in conjunction with quantitative data may provide a more in-depth understanding about the application of approaches in everyday clinical practice. This is a potential area for future research.

The majority of studies in this review used a range of clinician-reported outcome measures and included a variety of professional groups. If a more standardized approach was adopted by future studies, this would allow for easier comparison and meta-analysis.

\section{Review limitations}

The findings and conclusions of this review should be considered within the context of the limitations. A small number of studies were included, and for ease of comparison we only 
included studies written in English, which may have resulted in the exclusion of relevant studies written in other languages. All included studies were conducted in English-speaking high-income countries, so the results may not be applicable to broader international settings. Publication bias is also possible, and caution is required when interpreting the results due to their high degree of heterogeneity. Due to the lack of a comparison group in the studies, it is likely that extraneous variables could have influenced the findings.

\section{Conclusion}

We identified five relevant studies that reported the effects of medication-management training on mental health practitioners' knowledge, attitudes, and skills. The clinician outcomes significantly improved immediately following the training interventions; however, it is difficult to draw strong conclusions from the data due to the weaknesses associated with the designs of the studies. Future research in this area should aim to use validated standardized outcome measures, report the durability of improvements over a significant period of time following training, utilize a control group, and consider collecting qualitative data to explore clinicians' experiences of using the approaches in clinical practice.

\section{Disclosure}

The authors report no conflicts of interest in this work.

\section{References}

1. Weiden PJ, Kozma C, Grogg A, Locklear J. Partial compliance and risk of rehospitalization among California Medicaid patients with schizophrenia. Psychiatr Serv. 2004;55:886-891.

2. Valenstein M, Ganoczy D, McCarthy JF, Myra Kim H, Lee TA, Blow FC. Antipsychotic adherence over time among patients receiving treatment for schizophrenia: a retrospective review. J Clin Psychiatry. 2006;67: $1542-1550$.

3. Novick D, Haro JM, Suarez D, Perez V, Dittmann RW, Haddad PM. Predictors and clinical consequences of non-adherence with antipsychotic medication in the outpatient treatment of schizophrenia. Psychiatry Res. 2010;176:109-113.

4. Kemp R, Kirov G, Everitt B, Hayward P, David A. Randomised controlled trial of compliance therapy. 18-Month follow-up. Br J Psychiatry. 1998;172:413-419.

5. Maneesakorn S, Robson D, Gournay K, Gray R. An RCT of adherence therapy for people with schizophrenia in Chiang Mai, Thailand. J Clin Nurs. 2007;16:1302-1312.

6. Gray R, Leese M, Bindman J, et al. Adherence therapy for people with schizophrenia. European multicentre randomised controlled trial. $\mathrm{Br} J$ Psychiatry. 2006;189:508-514.

7. Gray R, White J, Schulz M, Abderhalden C. Enhancing medication adherence in people with schizophrenia: an international programme of research. Int J Ment Health Nurs. 2010;19:36-44.
8. Harris N, Lovell K, Day J, Roberts C. An evaluation of a medication management training programme for community mental health professionals; service user level outcomes: a cluster randomised controlled trial. Int J Nur Stud. 2009;46:645-652.

9. Byrne M, Deane F. Enhancing patient adherence: outcomes of medication alliance training on therapeutic alliance, insight, adherence, and psychopathology with mental health patients. Int J Ment Health Nurs. 2011;20:284-295.

10. Byrne MK, Deane FP, Coombs T. Nurse's beliefs and knowledge about medications are associated with their difficulties when using patient adherence strategies. J Ment Health. 2005;14:513-521.

11. Critical Appraisal Skills Programme (CASP). Making sense of evidence about clinical effectiveness. Available from: http://www.casp-uk.net/wpcontent/uploads/2011/11/CASP_Cohort_Appraisal_Checklist_14oct10. pdf. Accessed April 29, 2013.

12. Petticrew M, Roberts H. Systematic Reviews in the Social Sciences: A Practical Guide. Oxford: Blackwell; 2006.

13. Cohen J. Statistical Power Analysis for the Behavioral Sciences, 2nd ed. Hillsdale (NJ): Erlbaum; 1988.

14. Sharpe D. Of apples and oranges, file drawers and garbage: why validity issues in meta-analysis will not go away. Clin Psychol Rev. 1997;17: 881-901.

15. Moher D, Liberati A, Tetzlaff J, Altman DG; PRISMA Group. Preferred reporting items for systematic reviews and meta-analyses: the PRISMA statement. PLoS Med. 2009;6:e1000097.

16. Byrne M, Deane F, Lambert G, Coombs T. Enhancing medication adherence: clinician outcomes from the Medication Alliance training program. Aust NZ J Psychiatry. 2004;38:246-253.

17. Gray R, Wykes T, Gournay K. The effect of medication management training on community mental health nurse's clinical skills. Int J Nurs Stud. 2003;40:163-169.

18. Surguladze S, Timms P, David AS. Teaching psychiatric trainees 'compliance therapy.' Psychiatr Bull. 2002;26:12-15.

19. Byrne M, Willis A, Deane F, Hawkins B, Quinn R. Training inpatient mental health staff how to enhance patient engagement with medications: Medication Alliance training and dissemination outcomes in a large US mental health hospital. J Eval Clin Pract. 2010;16:114-120.

20. Vallis TM, Shaw BF, Dobson KS. The cognitive therapy scale: psychometric properties. J Consult Clin Psychol. 1986;54:381-385.

21. Haddock G, Devane S, Bradshaw T, et al. An investigation into the psychometric properties of the Cognitive Therapy Scale for Psychosis (CTSPsy). Behav Cogn Psychother. 2001;29:221-233.

22. Cartwright AKJ. The attitudes of helping agents towards the alcoholic client: the infuence of experience, support, training, and self-esteem. Br J Addict. 1980;75:413-431.

23. Gorman DM, Cartwright AKJ. Implications of using the composite and short versions of the Alcohol and Alcohol Problems Perception Questionnaire (AAPPQ). Br J Addiction. 1991;86:327-334.

24. Elsom S. The Therapeutic Optimism Scale. Melbourne: Monash University; 2002.

25. Wass V, Van der Vleuten C, Shatzer J, Jones R. Assessment of clinical competence. Lancet. 2001;357:945-949.

26. Morrison K. Developing reflective practice in higher degree students through a learning journal. Stud High Educ. 1996;21:317-332.

27. McGrath D, Higgins A. Implementing and evaluating reflective practice group sessions. Nurse Educ Pract. 2006;6:175-181.

28. Turner S, Beddoes L. Using reflective models to enhance learning: experiences of staff and students. Nurse Educ Pract. 2007;7: $135-140$. 
Nursing: Research and Reviews

Dovepress

\section{Publish your work in this journal}

Nursing: Research and Reviews is an international, peer-reviewed, open access journal publishing original research, reports, reviews and commentaries on all aspects of nursing and patient care. These include patient education and counselling, ethics, management and organizational issues, diagnostics and prescribing, economics and

resource management, health outcomes, and improving patient safety in all settings. The manuscript management system is completely online and includes a very quick and fair peer-review system. Visit http://www.dovepress.com/testimonials.php to read real quotes from published authors.

Submit your manuscript here: http://www.dovepress.com/nursing-research-and-reviews-journal 